\title{
openheart Normative blood pressure response to exercise stress testing in children and adolescents
}

\author{
Melanie M Clarke (10 , ,2 Diana Zannino, ${ }^{3}$ Natalie P Stewart, ${ }^{1}$ \\ Jonathan P Glenning, ${ }^{1,2}$ Salvador Pineda-Guevara, ${ }^{1}$ Jolien Kik, ${ }^{4,5}$ \\ Jonathan P Mynard, ${ }^{1,2,6}$ Michael M H Cheung ${ }^{1,2,4}$
}

To cite: Clarke MM, Zannino D, Stewart NP, et al. Normative blood pressure response to exercise stress testing in children and adolescents. Open Heart 2021;8:e001807. doi:10.1136/ openhrt-2021-001807

Received 6 August 2021 Accepted 22 September 2021

Check for updates

(c) Author(s) (or their employer(s)) 2021. Re-use permitted under CC BY-NC. No commercial re-use. See rights and permissions. Published by BMJ.

${ }^{1}$ Heart Research, Murdoch Children's Research Institute, Parkville, Victoria, Australia ${ }^{2}$ Department of Paediatrics, University of Melbourne, Parkville, Victoria, Australia ${ }^{3}$ Clinical Epidemiology and Biostatistics Unit, Murdoch Children's Research Institute, Parkville, Victoria, Australia ${ }^{4}$ Department of Cardiology, Royal Children's Hospital Melbourne, Melbourne, Victoria, Australia

${ }^{5}$ Medical Education, University of Groningen, Groningen, The Netherlands

${ }^{6}$ Biomedical Engineering, Faculty of Science, University of Melbourne, Melbourne, Victoria, Australia

Correspondence to Ms Melanie M Clarke; melanie. clarke@mcri.edu.au

\section{ABSTRACT}

Objective To describe normative values for blood pressure (BP) response to maximal exercise in children/ adolescents undergoing a treadmill stress test.

Methods From a retrospective analysis of medical records, patients who had undergone a Bruce protocol exercise stress test, with (1) normal cardiovascular system and (2) a body mass index percentile rank below $95 \%$ were included for analysis. Sex, age, height, weight, resting and peak heart rate, resting and peak systolic blood pressure (SBP), test duration, stage of Bruce protocol at termination, reason for undergoing the test and reason for termination of test were collected. Percentiles for exercise-induced changes in SBP were constructed by age and height for each sex with the use of quantile regression models.

Results 648 patients with a median age of 12.4 years (range 6-18 years) were included. Typical indications for stress testing were investigation of potential rhythm abnormalities, syncope/dizziness and chest pain and were deemed healthy by an overseeing cardiologist. Mean test duration was $12.6 \pm 2.2 \mathrm{~min}$. Reference percentiles for change in SBP by sex, age and height are presented. Conclusion The presented reference percentiles for the change in SBP for normal children and adolescents will have utility for detecting abnormally high or low BP responses to exercise in these age groups.

\section{INTRODUCTION}

Paediatric exercise testing is used in the clinical assessment of possible arrhythmias, dyspnoea, ischaemia and cardiac dysfunction, with blood pressure (BP) being a routine measure of the physiological response. While a moderate increase in BP with exercise is expected in children, normative values for treadmill exercise are not available. In adults, the normal BP response to exercise is well established $^{23}$ with a systolic value of $\geq 220 \mathrm{~mm}$ $\mathrm{Hg}$ considered an abnormally high exercise BP. ${ }^{4-6}$ Conversely, there is currently no definition of an excessive BP response to treadmill exercise for the paediatric population.

\section{Key questions}

What is already known about this subject?

- There are established normative values for treadmill exercise for adults. In children, normative blood pressure responses to treadmill exercise have not been established.

What does this study add?

- This study provides normative values and percentiles for systolic blood pressure (SBP) responses for treadmill exercise for male and female children and adolescents aged $6-18$ years old.

How might this impact on clinical practice?

- This study provides percentile tables for exercising SBP which can be used by clinicians in monitoring children and adolescents undergoing a Bruce protocol treadmill test.

Although the diagnostic significance of adult $\mathrm{BP}$ at rest and $\mathrm{BP}$ change with exercise are based on absolute cut-off values, resting paediatric BP values are typically classified by percentiles due to changes in normative values with growth and age. ${ }^{78}$ For example, a 10-year-old male standing at $135 \mathrm{~cm}$ would be considered hypertensive with a resting systolic blood pressure (SBP) of $114 \mathrm{~mm}$ $\mathrm{Hg}$, whereas a 20-year-old male would be considered hypertensive with a resting SBP of $130 \mathrm{~mm} \mathrm{Hg}{ }^{7}$ Therefore, the use of adult exercise cut-off values is likely to cause underdetection of excessive exercise BP responses in children. Under resting conditions, elevated BP is defined as $\geq 90$ th percentile, and hypertension is defined as $\mathrm{BP}$ of $\geq 95$ th percentile in children for their age, height and sex, with absolute cut-offs introduced at the age of 13 or 16 years of age (American and European guidelines, respectively). ${ }^{78}$ Accordingly, we suggest that an excessive BP response to treadmill exercise in the paediatric population should also be presented as percentiles, with the 90 th percentile defining 
elevated BP response and the 95th percentiles defining a hypertensive response.

Reference values for maximal treadmill exercise in normal healthy children are needed, noting that this form of stress test is the most commonly used in clinical settings. Thus far, the literature reporting normative paediatric cardiovascular response to exercise is predominately limited to cycle ergometer tests. ${ }^{910}$ To the best of our knowledge, BP percentiles for treadmill tests are limited, with the exception of submaximal stimuli ${ }^{2}$ and athletes, ${ }^{11}$ or they do not take into account height percentiles. ${ }^{12}$

The aim of this study was therefore to define normative reference values for maximal exercise BP response in children and adolescents for the standard Bruce treadmill stress test.

\section{METHODS}

Data were collected retrospectively. It was not possible to involve patients or the public in the design, conduct, reporting or dissemination plans of our research study.

Data were collected from pre-existing stress test results from patients who had undergone a routine standard Bruce protocol exercise stress test from 1990 to 2018 at Royal Children's Hospital. Typical indications for testing included chest pain, palpitations, dizziness and syncope but were considered normal after assessment by a qualified cardiologist. All tests were supervised by a cardiac technologist and medical staff.

\section{Exercise stress test}

After patient preparation and application of ECG electrodes in the supine position, an appropriately sized BP cuff was placed over the right upper arm. The patient lay in the supine position for approximately $10 \mathrm{~min}$ prior to a single resting auscultatory BP measurement. ${ }^{1}$ The cuff was inflated to approximately $20 \mathrm{~mm} \mathrm{Hg}$ above SBP, after which the pressure was slowly released from the cuff. Resting SBP and diastolic blood pressure (DBP) were recorded via the auscultatory method using a calibrated aneroid manometer. The patient was then moved to the treadmill where the
Bruce protocol was used, beginning at an incline grade of $10 \%$ and speed of $2.74 \mathrm{~km} /$ hour and increasing by $2 \%$ at $3 \mathrm{~min}$ intervals. The speed also increased with each change in inclination, until the patient reached volitional fatigue or completed the seventh and final stage (22\% grade and $9.65 \mathrm{~km} /$ hour). On completion of the test, the patient immediately returned to the supine position, where peak $\mathrm{BP}$ was measured. The reason for termination of exercise was recorded. Data were recorded and entered into an electronic database.

\section{Data analysis}

Patient records were assessed to ensure that they completed a standard Bruce protocol exercise stress test and that they had structurally and functionally normal cardiovascular systems. Additionally, the patient's body mass index (BMI) was compared against Centers for Disease Control and Prevention (CDC, Washington, DC, USA) BMI-for-age charts. Subjects with a BMI of $>95$ th percentile for their sex and age and subjects whose underlying symptomatology may have influenced BP response (ie, coarctation of the aorta) were excluded. Subjects with a resting BP reading that was considered hypertensive, $\geq 95$ th percentile based on age, sex and height, as defined by Flynn et al, ${ }^{7}$ were excluded. Where multiple exercise tests were performed by a particular patient, results from the first test were used. As an indicator of adequate effort, only data where the children had reached $85 \%$ of their age-predicted heart rate and were in a normal heart rhythm were included. The following variables were recorded: sex, age, height, weight, resting SBP, resting heart rate, peak SBP, peak heart rate, duration of test, stage of Bruce protocol, recorded reason for terminating the test and indication for the stress test. For the purpose of this study, we have only provided SBP reference values since measurement of DBP with exercise is unreliable in children. ${ }^{13-19}$

\section{Statistical analysis}

\section{Sample size justification}

At rest, a BP above the 95th percentile defines hypertension in children (up to the age of 13 or 16 years). ${ }^{78}$ In

\begin{tabular}{|c|c|c|c|}
\hline Variable & Total $(\mathrm{N}=648)$ & Female $(n=334)$ & Male $(n=314)$ \\
\hline Age (years) & $12.4(9.7-14.8)$ & $12.9(10-15)$ & $12.2(9.6-14.7)$ \\
\hline Weight percentile & $61.9(38.2-81.7)$ & $63.4(42.4-82.2)$ & $61.1(36.6-81.7)$ \\
\hline Height percentile & $65.1(38.1-84.1)$ & $65.4(38.3-82.8)$ & $64.8(37.6-84.6)$ \\
\hline BMI percentile & $58.4(31.6-77.2)$ & $62.2(37-80.2)^{\star}$ & $55.2(27.2-75.1)$ \\
\hline \multicolumn{4}{|l|}{ BMI category } \\
\hline Healthy & $619(82 \%)$ & 307 (82\%) & $312(83 \%)$ \\
\hline Overweight & $107(14 \%)$ & $57(15 \%)$ & $50(13 \%)$ \\
\hline Underweight & $27(4 \%)$ & $12(3 \%)$ & $15(4 \%)$ \\
\hline
\end{tabular}

Data presented as median (IQR).

${ }^{*} \mathrm{P}<0.001$ compared with males.

BMI, body mass index. 
this study, we defined percentiles in children and adolescents up to age 18 years. Using regression-based reference limits, a 95\% reference range (defining exercise hypertension), $90 \% \mathrm{CI}, 10 \%$ relative margin of error for the reference range and assuming a uniform distribution of ages, we found that a sample size of 377 was required (calculated via MedCalc software).

\section{Statistical methods}

Analyses were performed in Stata V.16 and R software V.4.0.1. Patient characteristics are presented for the whole sample and by sex. As per the reference ranges provided by Flynn et al, ${ }^{7}$ we constructed age, sex and height percentile-specific reference ranges using quantile regression to estimate the 5th, 10th, 50th, 90th and 95th percentile reference curves for the change in SBP, measured as the difference between SBP at rest and at peak exercise.

\section{RESULTS}

A total of 756 medical records were assessed $(50 \%$ male). Of these, 42 were excluded due to BMI of $>95$ th percentile, and 66 were excluded due to failure to reach sufficient exercise intensity of $\geq 85 \%$ of age-predicted maximum heart rate. This left 334 records for females, and 314 records for males. The median age was 13 years for females and 12 years for males (table 1). Most patients ( $\sim 80 \%$, for both males and females) had a healthy BMI (58th percentile) (table 1), with females having a higher BMI (62nd percentile) than males (55th percentile) $(\mathrm{p}<0.001)$.

The average test duration was $12.6 \pm 2.2 \mathrm{~min}$, with males having a longer average test duration compared with females $(p<0.001)$. The average increase in SBP from rest to peak exercise was $43 \pm 15 \mathrm{~mm} \mathrm{Hg}$ and did not differ between males and females $(\mathrm{p}=0.2)$ (table 2). The average resting heart rate was $83 \pm 16$ beats/min and increased to $195 \pm 9$ beats $/ \mathrm{min}$ at peak exertion (table 2 ).

Percentiles for female and male SBP changes in response to exercise are provided in tables 3 and 4 ,

\begin{tabular}{llll}
\hline Table 2 & & & \\
\hline Variable & Total (N=648) & Female (n=334) & Male (n=314) \\
\hline Resting SBP (mm Hg) & $101.2(10.0)$ & $101.1(10.3)$ & $101.4(9.8)$ \\
\hline Resting SBP percentile & $38.5(26.6)$ & $38.0(27.1)$ & $35.1(23.5)$ \\
\hline Peak SBP (mm Hg) & $144.3(18.2)$ & $143.5(16.7)$ & $145.2(19.6)$ \\
\hline Change in SBP (mm Hg) & $43.1(14.6)$ & $42.4(13.9)$ & $43.8(15.3)$ \\
\hline Resting DBP (mm Hg) & $58.9(7.6)$ & $59.2(7.9)$ & $38.7(7.3)$ \\
\hline Resting DBP percentile & $39.7(23.6)$ & $38.3(23.3)$ & $81.9(20.1)$ \\
\hline Resting HR (beats/min) & $83.1(15.6)$ & $84.2(15.5)$ & $194.7(9.7)$ \\
\hline Peak HR (beats/min) & $195.0(9.4)$ & $195.2(9.0)$ & $93.7(4.9)$ \\
\hline Percentage of predicted maximum HR & $93.9(4.7)$ & $94.1(4.6)$ & $13.3(2.4)^{\star}$ \\
\hline Test duration & $12.6(2.2)$ & $12.0(1.9)$ & $1(0 \%)$ \\
\hline Stage of Bruce protocol reached & & & $5(2 \%)$ \\
\hline 2 & $1(0 \%)$ & $0(0 \%)$ & $79(25 \%)$ \\
\hline 3 & $16(2 \%)$ & $11(3 \%)$ & $153(49 \%)$ \\
\hline 4 & $228(35 \%)$ & $149(45 \%)$ & $68(22 \%)$ \\
\hline 5 & $303(47 \%)$ & $150(45 \%)$ & $8(3 \%)$ \\
\hline 6 & $90(14 \%)$ & $22(7 \%)$ & $6(2 \%)$ \\
\hline 7 & $10(2 \%)$ & $2(1 \%)$ & $12(4 \%)$ \\
\hline Reason for termination & & & $293(93 \%)$ \\
\hline Adequate or complete time reached & $9(1 \%)$ & $3(1 \%)$ & $0(0 \%)$ \\
\hline Chest pain/dizziness/collapse & $54(8 \%)$ & $42(13 \%)$ & $282(84 \%)$ \\
\hline Fatigue/shortness of breath/sore body (chest)/anxiety/ & $575(89 \%)$ & $1(0 \%)$ & $2(1 \%)$ \\
\hline distress/poor treadmill coordination & $4(1 \%)$ & $4(1 \%)$ & \\
\hline Other & $2(0 \%)$ & & \\
\hline Sudden collapse & $4(1 \%)$ & & \\
\hline Unknown & & & \\
\hline
\end{tabular}

Data presented as mean (SD) or $\mathrm{N}(\%)$.

${ }^{*} \mathrm{P}<0.001$ compared with females.

DBP, diastolic blood pressure; HR, heart rate; SBP, systolic blood pressure. 
Table 3 Percentiles for female SBP response to exercise (ie, change from baseline pressure) by age and height $(n=334)$

\section{Height percentile}

\section{Change in SBP percentile}

$\begin{array}{llllllll}\text { Age Unit } & \text { P5 } & \text { P10 } & \text { P25 } & \text { P50 } & \text { P75 } & \text { P90 } & \text { P95 }\end{array}$ $\begin{array}{lllllllll}6 & \mathrm{~cm} & 106.9 & 108.6 & 111.6 & 115 & 118.6 & 121.9 & 123.9\end{array}$ $\begin{array}{llllllll}\text { in } & 42.1 & 42.8 & 43.9 & 45.3 & 46.7 & 48 & 48.8\end{array}$ $\begin{array}{llllllll}5 \text { th } & 16 & 17 & 18 & 20 & 21 & 22 & 23\end{array}$ $\begin{array}{llllllll}\text { 10th } & 18 & 18 & 19 & 20 & 21 & 21 & 22\end{array}$ $\begin{array}{llllllll}50 \text { th } & 33 & 34 & 35 & 36 & 37 & 38 & 39\end{array}$ $\begin{array}{llllllll}90 \text { th } & 38 & 39 & 42 & 44 & 47 & 49 & 50\end{array}$ $\begin{array}{llllllll}95 \text { th } & 42 & 43 & 44 & 46 & 48 & 49 & 50\end{array}$

$\begin{array}{lllllllll}7 & \mathrm{~cm} & 113.1 & 114.9 & 118.1 & 121.8 & 125.6 & 129.1 & 131.3\end{array}$ $\begin{array}{llllllll}\text { in } & 44.5 & 45.2 & 46.5 & 47.9 & 49.4 & 50.8 & 51.7\end{array}$ $\begin{array}{llllllll}\text { 5th } & 16 & 17 & 18 & 20 & 21 & 23 & 23\end{array}$ $\begin{array}{llllllll}\text { 10th } & 19 & 19 & 20 & 21 & 22 & 22 & 23\end{array}$ $\begin{array}{llllllll}50 \text { th } & 34 & 35 & 36 & 37 & 38 & 39 & 39\end{array}$ $\begin{array}{llllllll}90 \text { th } & 40 & 42 & 44 & 46 & 49 & 51 & 53\end{array}$ $\begin{array}{llllllll}95 \text { th } & 44 & 45 & 47 & 49 & 50 & 52 & 53\end{array}$ $\begin{array}{lllllllll}8 & \mathrm{~cm} & 118.5 & 120.5 & 123.9 & 127.8 & 131.9 & 135.6 & 137.9\end{array}$ $\begin{array}{llllllll}\text { in } & 46.7 & 47.5 & 48.8 & 50.3 & 51.9 & 53.4 & 54.3\end{array}$ $\begin{array}{llllllll}5 \text { th } & 17 & 17 & 19 & 20 & 21 & 23 & 23\end{array}$ $\begin{array}{llllllll}\text { 10th } & 20 & 20 & 21 & 22 & 22 & 23 & 24\end{array}$ $\begin{array}{llllllll}50 \text { th } & 35 & 36 & 37 & 38 & 39 & 40 & 40\end{array}$ $\begin{array}{llllllll}90 \text { th } & 42 & 44 & 46 & 49 & 51 & 53 & 55\end{array}$ $\begin{array}{llllllll}95 \text { th } & 47 & 48 & 49 & 51 & 53 & 54 & 55\end{array}$

$\begin{array}{lllllllll}9 & \mathrm{~cm} & 123.2 & 125.3 & 129 & 133.1 & 137.4 & 141.4 & 143.8\end{array}$ $\begin{array}{llllllll}\text { in } & 48.5 & 49.3 & 50.8 & 52.4 & 54.1 & 55.7 & 56.6\end{array}$ $\begin{array}{llllllll}\text { 5th } & 17 & 17 & 19 & 20 & 21 & 23 & 23\end{array}$ $\begin{array}{llllllll}10 \text { th } & 21 & 21 & 22 & 23 & 23 & 24 & 24\end{array}$ $\begin{array}{llllllll}\text { 50th } & 36 & 37 & 38 & 39 & 40 & 41 & 41\end{array}$ $\begin{array}{llllllll}90 \text { th } & 44 & 46 & 48 & 51 & 53 & 56 & 57\end{array}$ $\begin{array}{llllllll}95 \text { th } & 50 & 50 & 52 & 54 & 55 & 57 & 58\end{array}$

$\begin{array}{lllllllll}10 & \mathrm{~cm} & 127.5 & 129.8 & 133.7 & 138.2 & 142.8 & 147 & 149.6\end{array}$ $\begin{array}{llllllll}\text { in } & 50.2 & 51.1 & 52.6 & 54.4 & 56.2 & 57.9 & 58.9\end{array}$ $\begin{array}{llllllll}5 \text { th } & 17 & 17 & 19 & 20 & 22 & 23 & 24\end{array}$ $\begin{array}{llllllll}\text { 10th } & 22 & 22 & 23 & 23 & 24 & 25 & 25\end{array}$ $\begin{array}{llllllll}50 \text { th } & 37 & 37 & 38 & 39 & 41 & 42 & 42\end{array}$ $\begin{array}{llllllll}90 \text { th } & 47 & 48 & 50 & 53 & 55 & 58 & 59\end{array}$ $\begin{array}{llllllll}95 \text { th } & 52 & 53 & 55 & 56 & 58 & 60 & 60\end{array}$

$\begin{array}{lllllllll}11 & \mathrm{~cm} & 132.4 & 135 & 139.4 & 144.3 & 149.2 & 153.7 & 156.4\end{array}$ $\begin{array}{llllllll}\text { in } & 52.1 & 53.1 & 54.9 & 56.8 & 58.7 & 60.5 & 61.6\end{array}$ $\begin{array}{llllllll}\text { 5th } & 17 & 18 & 19 & 20 & 22 & 23 & 24\end{array}$ $\begin{array}{llllllll}10 \text { th } & 22 & 23 & 24 & 24 & 25 & 26 & 26\end{array}$ $\begin{array}{llllllll}50 \text { th } & 38 & 38 & 39 & 40 & 41 & 42 & 43\end{array}$ $\begin{array}{llllllll}90^{\text {th }} & 49 & 50 & 52 & 55 & 58 & 60 & 61\end{array}$ $\begin{array}{llllllll}95 \text { th } & 55 & 56 & 57 & 59 & 61 & 62 & 63\end{array}$

$\begin{array}{lllllllll}12 & \mathrm{~cm} & 139.2 & 142 & 146.5 & 151.5 & 156.4 & 160.8 & 163.5\end{array}$ $\begin{array}{llllllll}\text { in } & 54.8 & 55.9 & 57.7 & 59.6 & 61.6 & 61.6 & 64.4\end{array}$ $\begin{array}{llllllll}5 \text { th } & 17 & 18 & 19 & 20 & 22 & 23 & 24\end{array}$ Continued
Table 3 Continued

Height percentile

Change in SBP percentile

\begin{tabular}{lllllllll}
\hline Age & Unit & P5 & P10 & P25 & P50 & P75 & P90 & P95
\end{tabular}

$\begin{array}{llllllll}10 \text { th } & 23 & 24 & 24 & 25 & 26 & 27 & 27\end{array}$

$\begin{array}{llllllll}50 \text { th } & 39 & 39 & 40 & 41 & 42 & 43 & 44\end{array}$

$\begin{array}{llllllll}90 \text { th } & 51 & 52 & 55 & 57 & 60 & 62 & 63\end{array}$

$\begin{array}{llllllll}\text { 95th } & 57 & 58 & 60 & 61 & 63 & 65 & 66\end{array}$

$\begin{array}{lllllllll}13 & \mathrm{~cm} & 145.9 & 148.4 & 152.7 & 157.3 & 162 & 166.1 & 168.6\end{array}$ $\begin{array}{llllllll}\text { in } & 57.4 & 58.4 & 60.1 & 61.9 & 63.8 & 65.4 & 66.4\end{array}$ $\begin{array}{llllllll}5 \text { th } & 17 & 18 & 19 & 20 & 22 & 23 & 24\end{array}$ $\begin{array}{llllllll}\text { 10th } & 24 & 25 & 25 & 26 & 27 & 28 & 28\end{array}$ $\begin{array}{llllllll}\text { 50th } & 40 & 40 & 41 & 42 & 43 & 44 & 45\end{array}$ $\begin{array}{llllllll}90 \text { th } & 53 & 54 & 57 & 59 & 62 & 64 & 66\end{array}$ $\begin{array}{llllllll}95 \text { th } & 60 & 61 & 62 & 64 & 66 & 67 & 68\end{array}$

$\begin{array}{lllllllll}14 & \mathrm{~cm} & 149.7 & 152.1 & 156 & 160.5 & 164.9 & 168.9 & 171.3\end{array}$ $\begin{array}{llllllll}\text { in } & 58.9 & 59.9 & 61.4 & 63.2 & 64.9 & 66.5 & 67.4\end{array}$ $\begin{array}{llllllll}5 \text { th } & 17 & 18 & 19 & 20 & 22 & 23 & 24\end{array}$ $\begin{array}{llllllll}\text { 10th } & 25 & 25 & 26 & 27 & 28 & 28 & 29\end{array}$ $\begin{array}{llllllll}50 \text { th } & 40 & 41 & 42 & 43 & 44 & 45 & 46\end{array}$ $\begin{array}{llllllll}90 \text { th } & 55 & 57 & 59 & 62 & 64 & 66 & 68\end{array}$ $\begin{array}{llllllll}95 \text { th } & 62 & 63 & 65 & 66 & 68 & 70 & 71\end{array}$

$\begin{array}{lllllllll}15 & \mathrm{~cm} & 151.3 & 153.6 & 157.5 & 161.9 & 166.3 & 170.2 & 172.6\end{array}$ $\begin{array}{llllllll}\text { in } & 59.6 & 60.5 & 62 & 63.7 & 65.5 & 67 & 68\end{array}$ $\begin{array}{llllllll}\text { 5th } & 17 & 18 & 19 & 21 & 22 & 23 & 24\end{array}$ $\begin{array}{llllllll}10 \text { th } & 26 & 26 & 27 & 28 & 29 & 29 & 30\end{array}$ $\begin{array}{llllllll}50 \text { th } & 41 & 42 & 43 & 44 & 45 & 46 & 47\end{array}$ $\begin{array}{llllllll}90 \text { th } & 57 & 59 & 61 & 64 & 66 & 69 & 70\end{array}$

$\begin{array}{llllllll}95 \text { th } & 65 & 66 & 67 & 69 & 71 & 72 & 73\end{array}$

$\begin{array}{lllllllll}16 & \mathrm{~cm} & 151.9 & 154.3 & 158.2 & 162.6 & 166.9 & 170.9 & 173.2\end{array}$ $\begin{array}{llllllll}\text { in } & 59.8 & 60.7 & 62.3 & 64 & 65.7 & 67.3 & 68.2\end{array}$ $\begin{array}{llllllll}5 \text { th } & 17 & 18 & 19 & 21 & 22 & 23 & 24\end{array}$ $\begin{array}{llllllll}\text { 10th } & 27 & 27 & 28 & 29 & 30 & 30 & 31\end{array}$ $\begin{array}{llllllll}50 \text { th } & 42 & 43 & 44 & 45 & 46 & 47 & 47\end{array}$ $\begin{array}{llllllll}90 \text { th } & 60 & 61 & 63 & 66 & 68 & 71 & 72\end{array}$ $\begin{array}{llllllll}95^{\text {th }} & 67 & 68 & 70 & 72 & 73 & 75 & 76\end{array}$

$\begin{array}{lllllllll}17 & \mathrm{~cm} & 152.3 & 154.6 & 158.6 & 162.9 & 167.3 & 171.2 & 173.6\end{array}$ $\begin{array}{llllllll}\text { in } & 60 & 60.9 & 62.4 & 64.1 & 65.9 & 67.4 & 68.3\end{array}$ $\begin{array}{llllllll}\text { 5th } & 17 & 18 & 19 & 21 & 22 & 23 & 24\end{array}$ $\begin{array}{llllllll}\text { 10th } & 28 & 28 & 29 & 30 & 30 & 31 & 32\end{array}$ $\begin{array}{llllllll}50 \text { th } & 43 & 44 & 45 & 46 & 47 & 48 & 48\end{array}$ $\begin{array}{llllllll}90 \text { th } & 62 & 63 & 65 & 68 & 71 & 73 & 74\end{array}$ $\begin{array}{llllllll}\text { 95th } & 70 & 71 & 71 & 74 & 76 & 77 & 78\end{array}$

$\begin{array}{lllllllll}18 & \mathrm{~cm} & 152.5 & 154.8 & 158.8 & 163.1 & 167.5 & 171.4 & 173.8\end{array}$ $\begin{array}{llllllll}\text { in } & 60 & 61 & 62.5 & 64.2 & 65.9 & 67.5 & 68.4\end{array}$ $\begin{array}{llllllll}5 \text { th } & 17 & 18 & 19 & 21 & 22 & 23 & 24\end{array}$

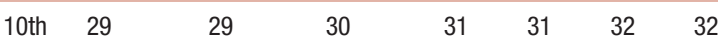
$\begin{array}{llllllll}50 \text { th } & 44 & 45 & 45 & 47 & 48 & 49 & 49\end{array}$ $\begin{array}{llllllll}90 \text { th } & 64 & 65 & 68 & 70 & 73 & 75 & 76\end{array}$ $\begin{array}{llllllll}\text { 95th } & 72 & 73 & 75 & 77 & 78 & 80 & 81\end{array}$

SBP, systolic blood pressure. 
Table 4 Percentiles for male SBP response to exercise (ie, change from baseline pressure) by age and height $(n=334)$

\section{Height percentile}

\section{Change in SBP percentile}

$\begin{array}{llllllll}\text { Age Unit } & \text { P5 } & \text { P10 } & \text { P25 } & \text { P50 } & \text { P75 } & \text { P90 } & \text { P95 }\end{array}$

$\begin{array}{lllllllll}6 & \mathrm{~cm} & 107.3 & 109.2 & 112.2 & 115.7 & 119.1 & 122.1 & 123.9\end{array}$

$\begin{array}{llllllll}\text { in } & 42.2 & 43 & 44.2 & 45.5 & 46.9 & 48.1 & 48.8\end{array}$

$\begin{array}{llllllll}\text { 5th } & 11 & 11 & 12 & 12 & 13 & 14 & 14\end{array}$

$\begin{array}{lllllllll}\text { 10th } & 16 & 16 & 17 & 18 & 19 & 19 & 20\end{array}$

$\begin{array}{llllllll}50 \text { th } & 28 & 29 & 30 & 31 & 32 & 32 & 33\end{array}$

$\begin{array}{llllllll}90 \text { th } & 36 & 37 & 38 & 40 & 42 & 44 & 45\end{array}$

$\begin{array}{llllllll}\text { 95th } & 41 & 43 & 45 & 47 & 50 & 52 & 53\end{array}$

$\begin{array}{lllllllll}7 & \mathrm{~cm} & 113.2 & 115.1 & 118.4 & 122 & 125.7 & 129 & 131\end{array}$

$\begin{array}{llllllll}\text { in } & 44.6 & 45.3 & 46.6 & 48 & 49.5 & 50.8 & 51.6\end{array}$

$\begin{array}{llllllll}\text { 5th } & 12 & 12 & 13 & 14 & 14 & 15 & 16\end{array}$

$\begin{array}{llllllll}10 \text { th } & 17 & 17 & 18 & 19 & 20 & 21 & 21\end{array}$

$\begin{array}{llllllll}50 \text { th } & 30 & 31 & 32 & 33 & 34 & 34 & 35\end{array}$

$\begin{array}{llllllll}90 \text { th } & 39 & 40 & 42 & 44 & 45 & 47 & 48\end{array}$

$\begin{array}{llllllll}\text { 95th } & 44 & 46 & 48 & 50 & 53 & 55 & 56\end{array}$

$\begin{array}{lllllllll}8 & \mathrm{~cm} & 118.8 & 120.8 & 124.3 & 128.1 & 132.1 & 135.7 & 137.8\end{array}$

$\begin{array}{llllllll}\text { in } & 46.8 & 47.6 & 48.9 & 50.4 & 52 & 53.4 & 54.3\end{array}$

$\begin{array}{llllllll}5 \text { th } & 13 & 14 & 14 & 15 & 16 & 16 & 17\end{array}$

$\begin{array}{llllllll}\text { 10th } & 18 & 19 & 19 & 20 & 21 & 22 & 22\end{array}$

$\begin{array}{llllllll}50 \text { th } & 32 & 33 & 34 & 35 & 36 & 36 & 37\end{array}$

$\begin{array}{llllllll}90 \text { th } & 42 & 43 & 45 & 47 & 49 & 51 & 52\end{array}$

$\begin{array}{llllllll}95 \text { th } & 47 & 49 & 51 & 53 & 56 & 58 & 59\end{array}$

$\begin{array}{lllllllll}9 & \mathrm{~cm} & 123.8 & 126 & 129.6 & 133.7 & 137.9 & 141.8 & 144.1\end{array}$

in $\quad \begin{array}{lllllll}48.7 & 49.6 & 51 & 52.7 & 54.3 & 55.8 & 56.7\end{array}$

$\begin{array}{llllllll}5 \text { th } & 15 & 15 & 16 & 16 & 17 & 18 & 18\end{array}$

$\begin{array}{llllllll}\text { 10th } & 19 & 20 & 21 & 21 & 22 & 23 & 24\end{array}$

$\begin{array}{llllllll}50 \text { th } & 34 & 35 & 36 & 37 & 38 & 39 & 39\end{array}$

$\begin{array}{llllllll}90 \text { th } & 46 & 47 & 48 & 50 & 52 & 54 & 55\end{array}$

$\begin{array}{llllllll}\text { 95th } & 50 & 52 & 54 & 56 & 59 & 61 & 62\end{array}$

$\begin{array}{lllllllll}10 & \mathrm{~cm} & 128.2 & 130.5 & 134.4 & 138.8 & 143.3 & 147.4 & 149.9\end{array}$

$\begin{array}{llllllll}\text { in } & 50.5 & 51.4 & 52.9 & 54.7 & 56.4 & 58 & 59\end{array}$

$\begin{array}{llllllll}5 \text { th } & 16 & 16 & 17 & 18 & 18 & 19 & 19\end{array}$

$\begin{array}{llllllll}\text { 10th } & 21 & 21 & 22 & 23 & 24 & 24 & 25\end{array}$

$\begin{array}{llllllll}50 \text { th } & 36 & 37 & 38 & 39 & 40 & 41 & 41\end{array}$

$\begin{array}{llllllll}90 \text { th } & 49 & 50 & 52 & 54 & 56 & 57 & 58\end{array}$

$\begin{array}{llllllll}95 \text { th } & 53 & 55 & 57 & 59 & 62 & 64 & 65\end{array}$

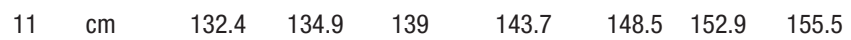

$\begin{array}{llllllll}\text { in } & 52.1 & 53.1 & 54.7 & 56.6 & 58.5 & 60.2 & 61.2\end{array}$

$\begin{array}{llllllll}\text { 5th } & 17 & 18 & 18 & 19 & 20 & 20 & 21\end{array}$

$\begin{array}{llllllll}\text { 10th } & 22 & 22 & 23 & 24 & 25 & 26 & 26\end{array}$

$\begin{array}{llllllll}50 \text { th } & 38 & 39 & 40 & 41 & 42 & 43 & 43\end{array}$

$\begin{array}{llllllll}90 \text { th } & 53 & 54 & 55 & 57 & 59 & 61 & 62\end{array}$

$\begin{array}{llllllll}\text { 95th } & 56 & 58 & 60 & 62 & 62 & 67 & 68\end{array}$

$\begin{array}{lllllllll}12 & \mathrm{~cm} & 137.3 & 139.9 & 144.3 & 149.3 & 154.4 & 159 & 161.9\end{array}$

$\begin{array}{llllllll}\text { in } & 54.1 & 55.1 & 56.8 & 58.8 & 60.8 & 62.6 & 63.7\end{array}$

$\begin{array}{llllllll}\text { 5th } & 18 & 19 & 19 & 20 & 21 & 22 & 22\end{array}$

$\begin{array}{llllllll}\text { 10th } & 23 & 23 & 24 & 25 & 26 & 27 & 27\end{array}$
Table 4 Continued

Height percentile

Change in SBP percentile

\begin{tabular}{llllllll}
\hline Age Unit & P5 & P10 & P25 & P50 & P75 & P90 & P95
\end{tabular}

$\begin{array}{llllllll}50 \text { th } & 40 & 41 & 42 & 43 & 44 & 45 & 45\end{array}$

$\begin{array}{llllllll}90 \text { th } & 56 & 57 & 59 & 61 & 62 & 64 & 65\end{array}$

$\begin{array}{llllllll}\text { 95th } & 59 & 61 & 63 & 65 & 68 & 70 & 71\end{array}$

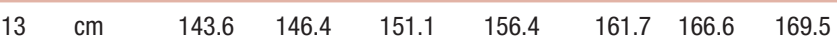

$\begin{array}{llllllll}\text { in } & 56.5 & 57.6 & 59.5 & 61.6 & 63.7 & 65.6 & 66.7\end{array}$

$\begin{array}{llllllll}5 \text { th } & 20 & 20 & 21 & 22 & 22 & 23 & 23\end{array}$

$\begin{array}{llllllll}10 \text { th } & 24 & 25 & 25 & 26 & 27 & 28 & 29\end{array}$

$\begin{array}{llllllll}50 \text { th } & 43 & 43 & 44 & 45 & 46 & 47 & 47\end{array}$

$\begin{array}{llllllll}90 \text { th } & 59 & 60 & 62 & 64 & 66 & 68 & 69\end{array}$

$\begin{array}{lllllllll}\text { 95th } & 62 & 64 & 66 & 68 & 71 & 73 & 74\end{array}$

$\begin{array}{lllllllll}14 & \mathrm{~cm} & 150.5 & 153.6 & 158.7 & 164.1 & 169.5 & 174.2 & 177\end{array}$

$\begin{array}{llllllll}\text { in } & 59.3 & 60.5 & 62.5 & 64.6 & 66.7 & 68.6 & 69.7\end{array}$

$\begin{array}{llllllll}5 \text { th } & 21 & 21 & 22 & 23 & 24 & 24 & 25\end{array}$

$\begin{array}{llllllll}\text { 10th } & 25 & 26 & 27 & 28 & 29 & 29 & 30\end{array}$

$\begin{array}{llllllll}50 \text { th } & 45 & 45 & 46 & 47 & 48 & 49 & 49\end{array}$

$\begin{array}{llllllll}\text { 90th } & 63 & 64 & 65 & 67 & 69 & 71 & 72\end{array}$

$\begin{array}{llllllll}95 \text { th } & 66 & 67 & 69 & 71 & 74 & 76 & 77\end{array}$

$\begin{array}{lllllllll}15 & \mathrm{~cm} & 156.7 & 159.8 & 164.8 & 170.1 & 175.3 & 179.8 & 182.4\end{array}$

$\begin{array}{llllllll}\text { in } & 61.7 & 62.9 & 64.9 & 67 & 69 & 70.8 & 71.8\end{array}$

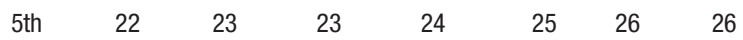

$\begin{array}{llllllll}\text { 10th } & 27 & 27 & 28 & 29 & 30 & 31 & 31\end{array}$

$\begin{array}{llllllll}50 \text { th } & 47 & 47 & 48 & 49 & 50 & 51 & 51\end{array}$

$\begin{array}{llllllll}90 \text { th } & 66 & 67 & 69 & 71 & 73 & 74 & 75\end{array}$

$\begin{array}{lllllllll}\text { 95th } & 69 & 70 & 72 & 74 & 77 & 79 & 80\end{array}$

$\begin{array}{lllllllll}16 & \mathrm{~cm} & 160.8 & 163.7 & 168.5 & 173.6 & 178.6 & 182.9 & 185.5\end{array}$ $\begin{array}{llllllll}\text { in } & 63.3 & 64.5 & 66.3 & 68.4 & 70.3 & 72 & 73\end{array}$

$\begin{array}{llllllll}5 \text { th } & 24 & 24 & 25 & 25 & 26 & 27 & 27\end{array}$

$\begin{array}{llllllll}\text { 10th } & 28 & 28 & 29 & 30 & 31 & 32 & 32\end{array}$

$\begin{array}{llllllll}50 \text { th } & 49 & 49 & 50 & 51 & 52 & 53 & 53\end{array}$

$\begin{array}{llllllll}90 \text { th } & 69 & 71 & 72 & 74 & 76 & 78 & 79\end{array}$

$\begin{array}{llllllll}\text { 95th } & 72 & 73 & 75 & 77 & 80 & 82 & 83\end{array}$

$\begin{array}{lllllllll}17 & \mathrm{~cm} & 163.1 & 165.8 & 170.4 & 175.3 & 180.2 & 184.5 & 187\end{array}$

$\begin{array}{llllllll}\text { in } & 64.2 & 65.3 & 67.1 & 69 & 70.9 & 72.6 & 73.6\end{array}$

$\begin{array}{llllllll}5 \text { th } & 25 & 25 & 26 & 27 & 27 & 28 & 28\end{array}$

$\begin{array}{llllllll}\text { 10th } & 29 & 30 & 30 & 31 & 32 & 33 & 33\end{array}$

$\begin{array}{llllllll}\text { 50th } & 51 & 51 & 52 & 53 & 54 & 55 & 55\end{array}$

$\begin{array}{llllllll}90 \text { th } & 73 & 74 & 76 & 78 & 79 & 81 & 82\end{array}$

$\begin{array}{lllllllll}\text { 95th } & 75 & 76 & 78 & 81 & 83 & 85 & 86\end{array}$

$\begin{array}{lllllllll}18 & \mathrm{~cm} & 164.2 & 166.9 & 171.3 & 176.2 & 181 & 185.3 & 187.8\end{array}$ $\begin{array}{llllllll}\text { in } & 64.7 & 65.7 & 67.5 & 69.4 & 71.3 & 72.9 & 73.9\end{array}$ $\begin{array}{llllllll}5 \text { th } & 26 & 27 & 27 & 28 & 29 & 29 & 30\end{array}$ $\begin{array}{llllllll}\text { 10th } & 30 & 31 & 32 & 33 & 33 & 34 & 35\end{array}$ $\begin{array}{llllllll}50 \text { th } & 53 & 53 & 54 & 55 & 56 & 57 & 57\end{array}$ $\begin{array}{llllllll}90 \text { th } & 76 & 77 & 79 & 81 & 83 & 85 & 86\end{array}$

$\begin{array}{llllllll}\text { 95th } & 78 & 79 & 81 & 84 & 86 & 88 & 89\end{array}$

SBP, systolic blood pressure. 
respectively. The changes in $\mathrm{BP}$ on exertion varied with age and height. Increases in SBP were higher in males than in females of the same age.

\section{DISCUSSION}

In adults, BP reference values are used to predict increased risk of morbidity and mortality, and there is evidence that exercise BP is superior for this purpose compared with resting $\mathrm{BP} .^{20-25}$ However, normative values for BP response to treadmill exercise in children and adolescents have not been available to date. It is currently unknown whether or not elevated exercise BP in children has the same predictive risk valu as that of adults. However, longitudinal studies of resting BP have found high BP in childhood tracks into adulthood. ${ }^{26}$ The normative values provided in this study will allow clinicians and researchers to identify those children with excessive increases in BP and follow them longitudinally and gain a better understanding of using exercise $\mathrm{BP}$ as a predictive risk assessment in the paediatric population. In addition, $\mathrm{BP}$ responses in conditions affecting the aortic arch such as repaired coarctation, transposition of the great arteries following arterial switch, Williams syndrome and many others can now be considered against normative data.

The majority of reference papers in the literature focus on cardiovascular response to cycle ergometers, and as such, reference values for treadmill testing are limited, ${ }^{9} 10$ with the exception of submaximal stimuli ${ }^{11}$ and athletes. ${ }^{9}$ Sasaki $e t a l^{12}$ recently published percentiles for children undergoing exercise on treadmills; their findings were based on a modified Bruce protocol and did not account for height or resting BP in their percentiles. ${ }^{12}$ Understanding BP response on a cycle ergometer is useful, especially as cycle ergometers are cheaper and quieter and require less space for exercise labs. ${ }^{1}$ Additionally, cycle ergometers are better for individuals with weight-bearing limitations ${ }^{27}$; however, individuals tend to reach muscular fatigue before reaching volitional fatigue. ${ }^{1}$ Conversely, volitional fatigue is reached first with treadmill exercise resulting in a maximal oxygen consumption approximately $10 \%$ greater than that of cycling. ${ }^{128}$ Therefore, if the purpose of the stress test is to reach volitional fatigue, treadmill exercise will be more appropriate for diagnostic purposes and determination of functional capacity in children. ${ }^{1}$ An additional benefit of treadmill exercise is that most individuals are familiar with the mechanics of walking from a very young age, whereas the biomechanics of cycling and maintaining cadence are not as familiar to all children. ${ }^{1}$ Furthermore, since treadmills are the most common apparatus for exercise testing in children, ${ }^{1}$ the reference values described herein will aid clinicians in determining normal and abnormal BP responses. Previously, a lack of current reference values meant diagnostic, prognostic and therapeutic decisions were currently based on subjective clinical experience. With this in mind, there is little consensus on what is appropriate for paediatric BP response to exercise, making clinical decisions difficult even with subjective experience. The data provided in this study can now be referenced to determine appropriate $\mathrm{BP}$ response and identify patients who may need further consultation in determining whether masked hypertension is present.

In young adults (20-29 years), the average change in SBP from rest to maximal exercise is $53 \pm 19 \mathrm{~mm} \mathrm{Hg}$ for males and $46 \pm 17 \mathrm{~mm} \mathrm{Hg}$ for females. ${ }^{29}$ Our study indicated excellent continuity with these findings, given that older adolescents (aged 18 years) had an average SBP response (50th percentile) of $55 \mathrm{~mm} \mathrm{Hg}$ for males and $47 \mathrm{~mm} \mathrm{Hg}$ for females. In adults, a single absolute BP cut-off of $220 \mathrm{~mm} \mathrm{Hg}$ has been suggested for defining exercise hypertension, although Schultz $e t a l^{30}$ noted that an exaggerated $\mathrm{BP}$ response is often expressed as a sex-dependent cut-off value at the 90th or 95th percentile (SBP; $\sim 210 \mathrm{~mm} \mathrm{Hg}$ for males and $190 \mathrm{~mm} \mathrm{Hg}$ for females). For adolescents $\geq 13$ years of age, single cutoff values have been used to define resting hypertensive status. ${ }^{7}$ However, in children, the percentile approach is more appropriate than a single cut-off, given the physiological and stature changes that continue during development. The 95th percentile of BP changes in 18 year olds adults (males: 78-89 mm Hg, females; 72-81 $\mathrm{mm} \mathrm{Hg}$, females, immediately post-exercise in supine position) were relatively similar to the changes seen in 20-29 year-olds ( 88 for males and $70 \mathrm{~mm} \mathrm{Hg}$ for females, measured during exercise stress test, calculated as difference between absolute 95th percentile and group average resting $\mathrm{BP}$ ) indicating that the use of percentiles is more appropriate up to the age of 18 for exercise BP. ${ }^{29}$

In the current study, percentiles were presented for all ages (6-18years). The European guidelines for management of arterial hypertension noted that while there are recommended cut-off values for exercise $\mathrm{BP}$, a single value does not take into account variations in pre-exercise BP, age, sex, arterial stiffness and obesity status. ${ }^{31}$ The normative values presented in this study took into account key individual characteristics, including age, sex, height and pre-exercise BP (by presenting change in SBP instead of peak exercise $\mathrm{BP})$.

\section{Study limitations and strengths}

The age distribution was approximately bell-shaped and hence was not uniformly distributed; however, this reflects the typical ages that children present for this form of testing. Reflecting the ethnic mix of this area of Australia, participants were mostly Caucasian (European and Mediterranean) but included children of Asian and Middle Eastern descent as well as other ethnic groups. Therefore, these data may not be representative of other ethnicities or regions. Data were collected using standard auscultatory methods ${ }^{1}$; while some centres may prefer oscillometric devices, to the best of our knowledge, no oscillometric devices have been validated for exercise BP measurement in the paediatric population. Since data were collected over two decades, the personnel involved in supervising the test inevitably varied. All BP 
measurements were taken by a cardiologist with at least 5 years' experience and using standard techniques.

As with all forms of exercise testing to assess peak ability, these depend on the volition of the child to perform. The strength of this study was the inclusion criteria that subjects had to reach a heart rate of at least $85 \%$ of maximum age-predicted heart rate as an indicator of adequate effort. We excluded obese individuals from this study, as these subjects tend to have increased $\mathrm{BP}$ that could skew the change in BP from rest to exercise, thus altering normal percentiles. ${ }^{7}$ Using these references values, it will now be possible to determine if children with obesity have an abnormal SBP response to exercise. Additionally, it will be possible to determine if other chronic pathological conditions, such as vascular and renal diseases, produce an exaggerated BP response with exercise.

\section{CONCLUSION}

We have described normative values of SBP response to maximal exercise ( $\geq 85 \%$ of age-predicted heart rate max) for a treadmill stress test in children and adolescents. These data will enable clearer identification of abnormal $\mathrm{BP}$ response and improve cardiovascular risk assessment in children.

\section{Twitter Melanie M Clarke @clark_eey}

Contributors MMHC, JPM and JK conceptualised and designed the study, provided intellectual feedback, and reviewed and revised the manuscript. JPG and NPS designed the data collection instruments, assisted in data collection, and reviewed and revised the manuscript. SP-G assisted in data collection and reviewed and revised the manuscript. DZ provided statistical expertise in generating normative value percentiles and reviewed and revised the manuscript. MMC helped conceptualise and design the study, designed the data collection instruments, coordinated and supervised data collection, drafted the manuscript, and reviewed and revised the manuscript. All authors approved the final manuscript as submitted and agreed to be accountable for all aspects of the work.

Funding The Heart Research Group is supported by the Victorian Government's Operational Infrastructure Support Program, RCH 1000 and Big W. The support from the funders had no role in the design and concept of this study.

Competing interests None declared.

Patient consent for publication Not applicable.

Ethics approval The Royal Children's Hospital (RCH) Human Research Ethics Committee.

\section{Provenance and peer review Not commissioned; externally peer reviewed.}

Data availability statement All data relevant to the study are included in the article or uploaded as supplemental information.

Open access This is an open access article distributed in accordance with the Creative Commons Attribution Non Commercial (CC BY-NC 4.0) license, which permits others to distribute, remix, adapt, build upon this work non-commercially, and license their derivative works on different terms, provided the original work is properly cited, appropriate credit is given, any changes made indicated, and the use is non-commercial. See: http://creativecommons.org/licenses/by-nc/4.0/.

\section{ORCID iD}

Melanie M Clarke http://orcid.org/0000-0001-6926-8812

\section{REFERENCES}

1 Paridon SM, Alpert BS, Boas SR, et al. Clinical stress testing in the pediatric age group: a statement from the American Heart Association council on Cardiovascular Disease in the Young, committee on Atherosclerosis, Hypertension, and Obesity in Youth. Circulation 2006;113:1905-20.

2 Turley KR, Wilmore $\mathrm{JH}$. Cardiovascular responses to treadmill and cycle ergometer exercise in children and adults. J Appl Physiol 1997;83:948-57.

3 Myers J, Prakash M, Froelicher V, et al. Exercise capacity and mortality among men referred for exercise testing. $N$ Engl $J$ Med 2002;346:793-801.

4 Takase B. Exercise stress testing as the significant clinical modality for management of hypertension. Hypertens Res 2012;35:706-7.

5 Pescatello LS, Franklin BA, Fagard R, et al. Exercise and hypertension. Med Sci Sports Exerc 2004;36:533-53.

6 Daida H, Allison TG, Squires RW, et al. Peak exercise blood pressure stratified by age and gender in apparently healthy subjects. Mayo Clin Proc 1996;71:445-52.

7 Flynn JT, Kaelber DC, Baker-Smith CM, et al. Clinical practice guideline for screening and management of high blood pressure in children and adolescents. Pediatrics 2017;140:e20171904.

8 Lurbe E, Agabiti-Rosei E, Cruickshank JK, et al. 2016 European Society of hypertension guidelines for the management of high blood pressure in children and adolescents. J Hypertens 2016;34:1887-920.

9 Hacke C, Weisser B. Reference values for exercise systolic blood pressure in 12- to 17-year-old adolescents. Am J Hypertens 2016;29:747-53.

10 Wanne OP, Haapoja E. Blood pressure during exercise in healthy children. Eur J Appl Physiol Occup Physiol 1988;58:62-7.

11 Szmigielska K, Szmigielska-Kapłon A, Jegier A. Blood pressure response to exercise in young athletes aged 10 to 18 years. Appl Physiol Nutr Metab 2016:41:41-8.

12 Sasaki T, Kawasaki Y, Takajo D, et al. Blood pressure response to treadmill cardiopulmonary exercise test in children with normal cardiac anatomy and function. J Pediatr 2021;233:169-74.

13 O'Sullivan J, Allen J, Murray A. A clinical study of the Korotkoff phases of blood pressure in children. J Hum Hypertens 2001;15:197-201.

14 Rosner B, Polk BF. The implications of blood pressure variability for clinical and screening purposes. J Chronic Dis 1979;32:451-61.

15 Biro FM, Daniels SR, Similo SL, et al. Differential classification of blood pressure by fourth and fifth Korotkoff phases in school-aged girls. The National Heart, Lung, and Blood Institute Growth and Health Study. Am J Hypertens 1996;9:242-7.

16 Londe S, Klitzner TS. Auscultatory blood pressure measurement-effect of pressure on the head of the stethoscope. West $J$ Med 1984;141:193-5.

17 Londe S. Fifth versus fourth Korotkoff phase. Pediatrics 1985;76:460-1.

18 Washington RL, Bricker JT, Alpert BS, et al. Guidelines for exercise testing in the pediatric age group. from the Committee on atherosclerosis and hypertension in children, Council on Cardiovascular Disease in the Young, the American Heart Association. Circulation 1994;90:2166-79.

19 Uhari M, Nuutinen M, Turtinen J, et al. Pulse sounds and measurement of diastolic blood pressure in children. Lancet 1991;338:159-61.

20 Filipovský J, Ducimetière P, Safar ME. Prognostic significance of exercise blood pressure and heart rate in middle-aged men. Hypertension 1992;20:333-9.

21 Mundal R, Kjeldsen SE, Sandvik L, et al. Exercise blood pressure predicts cardiovascular mortality in middle-aged men. Hypertension 1994;24:56-62.

22 Mundal R, Kjeldsen SE, Sandvik L, et al. Exercise blood pressure predicts mortality from myocardial infarction. Hypertension 1996;27:324-9.

23 Kjeldsen SE, Mundal R, Sandvik L, et al. Supine and exercise systolic blood pressure predict cardiovascular death in middle-aged men. J Hypertens 2001;19:1343-8.

24 Kokkinos P. Cardiorespiratory fitness, exercise, and blood pressure. Hypertension 2014;64:1160-4.

25 Jae SY, Franklin BA, Choo J, et al. Exaggerated exercise blood pressure response during treadmill testing as a predictor of future hypertension in men: a longitudinal study. Am J Hypertens 2015;28:1362-7.

26 Theodore RF, Broadbent J, Nagin D, et al. Childhood to earlymidlife systolic blood pressure trajectories: early-life predictors, effect modifiers, and adult cardiovascular outcomes. Hypertension 2015;66:1108-15.

27 Pina IL, Balady GJ, Hanson P, et al. Guidelines for clinical exercise testing laboratories. A statement for healthcare professionals from the Committee on Exercise and Cardiac Rehabilitation, American Heart Association. Circulation 1995;91:912-21. 
28 LeMura LM, von Duvillard SP, Cohen SL, et al. Treadmill and cycle ergometry testing in 5- to 6-year-old children. Eur J Appl Physiol 2001;85:472-8.

29 Sabbahi A, Arena R, Kaminsky LA, et al. Peak blood pressure responses during maximum cardiopulmonary exercise testing: Reference standards from FRIEND (Fitness Registry and the Importance of Exercise: A National Database). Hypertension 2018;71:229-36.
30 Schultz MG, La Gerche A, Sharman JE. Blood pressure response to exercise and cardiovascular disease. Curr Hypertens Rep 2017;19:89.

31 Williams B, Mancia G, Spiering W, et al. 2018 practice guidelines for the management of arterial hypertension of the European Society of Cardiology and the European Society of Hypertension. $J$ Hypertens 2018;36:2284-309. 